\title{
The Immense Impact of CCR5 Single-Nucleotide Polymorphisms (SNPs)
}

\author{
Tatiana Hillman \\ Biotechnology, TheLAB INC., Los Angeles, California, United States \\ Corresponding Author: First Author ${ }^{1}$ \\ Email':thillman@usc.edu
}

\begin{abstract}
C-C Chemokine receptor type 5 (CCR5) is expressed on the CD4 T cell surface where CXCR4 and CCR5 expressions are controlled differently during the activation of $T$ cells and with the binding of interleukin type-2 (IL-2). IL-2 can upregulate CCR1 and CCR2 in CD45R01 T cells and increase the T lymphocyte chemotaxis toward CC-chemokines. CD4+ T cells are either apart of the T helper 1 or Th1 lymphocytes that release interferon gamma (IFNy) and lymphotoxin that provide cellular immunity to internal pathogens and T helper cells type 2 (Th2), which secrete interleukins 4 and 5 (IL-4 and IL-5). IL-4 and IL-5 cause an allergic and humoral immune response to parasites. Th2 lymphocytes use CCR3 chemokine receptors. CCR5 and CXCR3 chemokine receptors are specific for CD4+ Th1 and Th2 lymphocytes. CCR5 $\triangle 32$ is a 32-base-pair deletion of the CCR5 gene. CCR5 is a co-receptor for the entrance of the human immunodeficiency virus-1 (HIV-1). CCR5 32 creates a malfunction of the CCR5 protein that can prevent HIV-1 infection. However, the CCR5 32 32-base-pair deletion is not prevalent and predominant in many populations worldwide and there also exist more genetic variations of CCR5 known as CCR5-SNPs. An alternative polymorphism was identified based on the CCR5 gene that was identified as a $A$ to $G(A / G)$ point mutation. This point mutation is located at the 59029 locus on the promoter that lowers the expression rate of CCR5. Gurdol et al. found activity at the promoter of CCR5/590029G was $45 \%$ lesser than the CCR5/59029A. The genetic variations of CCR5 SNPs also include: $2459 \mathrm{G}>$ SNP of CCR5, C101X, CCR5 gene position at -2273 , and the A to G SNP mutation found in two South African blacks. Therefore, more studies are needed to find more and varied chemokine polymorphisms that are present in many diverse populations in the world. The aim of this literature review is to describe the immense impact of CCR5 SNP mutations on viral infection susceptibility, on the pathogenesis of chronic conditions, to endorse the increased discovery of more novel CCR5 SNPs, and to show the significant potential of anti-CCR5 therapies to treat multiple diseased conditions.
\end{abstract}

Keywords: Single-Nucleotide Polymorphisms, Point Mutation, Chemokines, T-cells, Viral Infection, Tumorigenesis, T-cell Surface, Immune Response 


\section{INTRODUCTION}

Cytotoxic T cells, NK cells, dendritic cells (DCs), and macrophages assist with the immune response to antigens, pathogen-associated molecular patterns (PAMPs), and danger associated with molecular patterns (DAMPs). Chemokines help organize and attract these immune cells to sites of inflammation by interacting with receptors of these immune cells. The C-C chemokine receptor type 5 ,

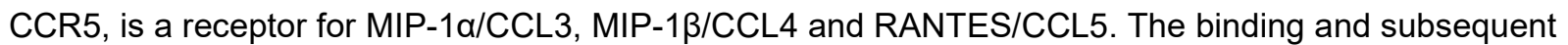
intracellular signaling of CCR5 to these ligands activates many transcription factors to initiate immune cell activation. This CCR5/ligands axis migrates immune responses to microbes, microbiotic small molecules, and to antigens. An alternative polymorphism was identified based on the CCR5 gene that was identified as a $A$ to $G(A / G)$ point mutation. This point mutation is located at the 59029 locus on the promoter that lowers the expression rate of CCR5. Activity at the promoter of CCR5/590029G was $45 \%$ lesser than the CCR5/59029A [11]. It was found that individuals homozygous for CCR5/590029A produce more CCR5 at the cell surface in CD4 immune T cells than heterozygotes with this genotype. Therefore, people who have the CCR5/590029-G/G genotype exhibit lesser responses to inflammation than CCR5/590029-A/A genotype carriers [1].

CCR5 $\triangle 32$ is a 32 -base-pair deletion of the CCR5 gene. CCR5 32 creates a malfunction of the CCR5 protein that can prevent HIV-1 infection. CCR5 is a co-receptor for the entrance of the human immunodeficiency virus-1 (HIV-1). CCR5 $\Delta 32$ alleles are mainly found in 10 to $15 \%$ of persons from European descent [2]. A study of Whites in the U.S confirmed the recovery from HBV was possible through the gene encoding CCR5 $\Delta 32$. People with the malfunctional receport of CCR5 $\Delta 32$ were twice as likely to survive and recover from HBV infection. Chemokines are characterized as low-molecular weight proteins that assist with the transport of immune cells during inflammation in an immune response. Chemokines bind to $G$ protein-coupled seven-transmembrane domain receptors to guide immune cells during an inflammatory response. Many $G$ protein-coupled receptor proteins are expressed on many cell type surfaces. The chemokine receptor type 5 (CCR5) identifies and then binds CCL5, CCL4, CCL3, and CCL8 chemokine ligands. CCR5 genes are located on 3p21 and shape into a cluster with other chemokine receptors of genes. CCR5 forms into a cluster that includes CCR2 and consists of CCL2, CCL8, and CCL13 ligands. Many variations in the genome of chemokine receptor gene loci can control the susceptibility of individuals to infectious and inflammatory diseases.

The Japanese Encephalitis Virus causes acute encephalitis syndrome or AES. Japanese Encephalitis or JE was the main cause of AES cases in 2010 in parts of north India [3]. The JE vaccine eliminated many cases of JE. JE has a single stranded RNA, ssRNA, as its viral genome. JE is a part of the Flaviviridae family of viruses that becomes transmitted through a female mosquito vector. Many people infected by JE lack or show mild symptoms. A JEV infection can transform into encephalitis. Because of JE, $45 \%$ of cases induce permanent neurological damage, and $18 \%$ of JE cases become terminal with a fatal prognosis [3]. Cytokines that are pro-inflammatory such as tumor necrosis factor of TNF-alpha, and interleukin (IL)-1-Beta, and chemokines such as CXCL-8, and monocyte chemoattractant protein (MCP)-1 in CCL-2 yielded after JEV infection allows the influx of the virus with monocytes of inflammation into the central nervous system. C-C chemokine receptor 5 or CCR5 plays a major role in the immune response against the West Nile Virus, which is also a member of the flavivirus family and causes encephalitis. By ablating CCR5 proved to weaken the symptoms and ultimate progression into JE. Studies have shown damaged neurons can release danger associated with molecular patterns or DAMP that lead into effects of neuro-inflammation. A high mobility box protein (HGB)-1 is a type of DAMP that intervenes in the neuroinflammatory effects of viral encephalitis transmitted by ticks [3]. 
Many other variations in the CCR5 gene can alter the susceptibility of people to HIV-1 and disease [4]. For example, the CCR2-V64I (rs1799864) polymorphism has an adenine changed into guanine at the 190 position of CCR2. The CCR2-V64I (rs1799864) polymorphism changes a valine (V) amino acid into an isoleucine (I) at the position of 64 at the first domain of the CCR2 protein. By deleting 32 base pairs form exon 1 of the CCR5 gene results in a frame shift change to the CCR5 gene at position 185 lessens the expression of the CCR5 protein and receptor. This 32-bp deletion is located on the extracellular loop of the protein receptor. THe CCR5 32-bp deletion and mutation is dominant and polymorphic in many ethnic populations and groups. Therefore, analyzing the CCR5 $\Delta 32$ mutation can guide us to improve the understanding of the most significant mechanisms necessary for chronic infections caused by microbes, diseases of the immune system, and autoimmune disorders [5].

Single nucleotide polymorphisms (SNPs) mediate the expression of these inflammatory mediators and chemokine receptors. SNPs located in the promoter and introns of TNFA, IL1B, CCL2, and CXCL8 affect and alter the levels of chemokines and cytokines. SNPs in the promoters of 32-base pair mutation of deletion of the CCR5 gene change the expression of CCR5. Most evidence includes studies of the CCR5 $\triangle 32$ polymorphism, which is a 32-bpd deletion in the CCR5 gene locus. A stop codon is introduced early and produces a malfunctioning receptor. Deng et al. studied and confirmed CCR5 as a co-receptor that interacts with the HIV-1 virus [3]. Huang et al. discovered that individuals homozygous for the CCR5 $\Delta 32$ mutation were more immune to HIV-1 infection [3I].

CCR5, CCR2, and stromal-derived factor 1 (SDF1) can regulate the individual susceptibility to HIV-1 infection and the progression of the disease. The effects of these gene polymorphisms with individual susceptibility to HIV-1 and AIDS is still controversial. Homozygous people with the CCR5$\Delta 32 / \Delta 32$ mutation are resistant to HIV-1 infection in many different ethnic populations and groups. Several individuals have been discovered to carry the CCR5- $\triangle 32 / \Delta 32$ genotype and were HIV- 1 positive [6]. The CCR5- $\Delta 32 / \Delta 32$ genotype is distributed across many ethnic groups. CCR5- $\Delta 32 / \Delta 32$ is observed in high frequency within the Caucasian population but in lower frequency within the Asian and African ethnic populations. In Cameroon no CCR5- $\Delta 32 / \Delta 32$ alleles are shown within the ethnic groups. Chemokines are small molecules that induce inflammatory responses of cytokines. Cysteine-cysteine (CC) chemokine receptor type 5 is a protein that is encoded in the CCR5 gene and localized in chromosome 3p.21-3p24. The open reading frame (ORF) containing a 32-base pair deletion in the CCR5 gene is more expressed and distributed throughout the Caucasian population. It is termed the CCR5 delta32 mutation. People homozygous for CCRD32 alleles can not express the functional protein of CCR5. Heterozygotes of the CCR5D32 allele express a limited functioning receptor than wild-type people homozygous for this allele. The CCR5D32 deletion and mutation affects the progression of autoimmune diseases and infections by controlling the inflammatory response.

In 2000 a research study noted the variation in HIV serotypes ranged from $0 \%$ to $18 \%$ in ethnic groups in Cameroon [6]. The HIV-AIDS committee reported a variation in the prevalence of HIV in different villages of Cameroon. In the western region where the Bamileke people live exhibit the lowest prevalence of HIV with 2.3\% [6]. Identifying the CCR2-64I, CCR5- $\triangle 32$, CCR5 promoter 59029 A/G and SDF1-3'A allelic mutants and analyzing their distribution can elucidate our understanding of HIV-1 infection when providing clinical decisions.CCR2-V64I polymorphisms can not produce the transmission of HIV-1; however, research studies documents that patients with the mutation in the $64 \mathrm{I}$ allele survive and have an improved prognosis after their viral infection. CCR5-D32 and CCR2-V64I polymorphisms are novel candidates for continued research of many diseases such as disorders of the circulatory system and neoplasms. However, for difficult and more chronic diseases, the study of polymorphisms in the clinical setting maintains inconclusivity and many studies continue to exhibit negative and conflicted 
results. For people of European descent, the CCR5-D32 and CCR2-V64I polymorphisms are highly prevalent in the European population.

For the African, Native American, and Asian populations the CCR5-D32 allele originated from a mutation in northern Europe thousands of years ago. The CCR2-V64I polymorphism is more commonly dispersed in the ethnic groups and populations globally [4]. Brazil is more racially diverse than many other countries in the world. Lopes et al. (2014) examined the ancestry and genetic drift of people in the Piauí State in northeastern Brazil. They used 46 identity ancestry markers. The population included $60 \%$ European, $18.5 \%$ idigenous peoples, and $21.5 \%$ African [4]. The distribution of the CCR5-D32 and CCR2-64I polymorphisms in varied regions of Brazil have not been studied. There is a high variability genetically in different populations globally that may serve to elucidate the issues of these research study types. More studies are needed that can confirm the varied chemokine polymorphisms from different populations [4]. There is a lack of data for African populations concerning host genetic polymorphisms preventing HIV-1 infection or delaying the progression of HIV into AIDS [6]. Therefore, this review will provide evidence for the impact of finding new and novel polymorphisms of CCR5 that can amplify the discovery of additional anti-CCR5 therapies for infectious diseases and chronic health disorders.

\section{Survey Methodology}

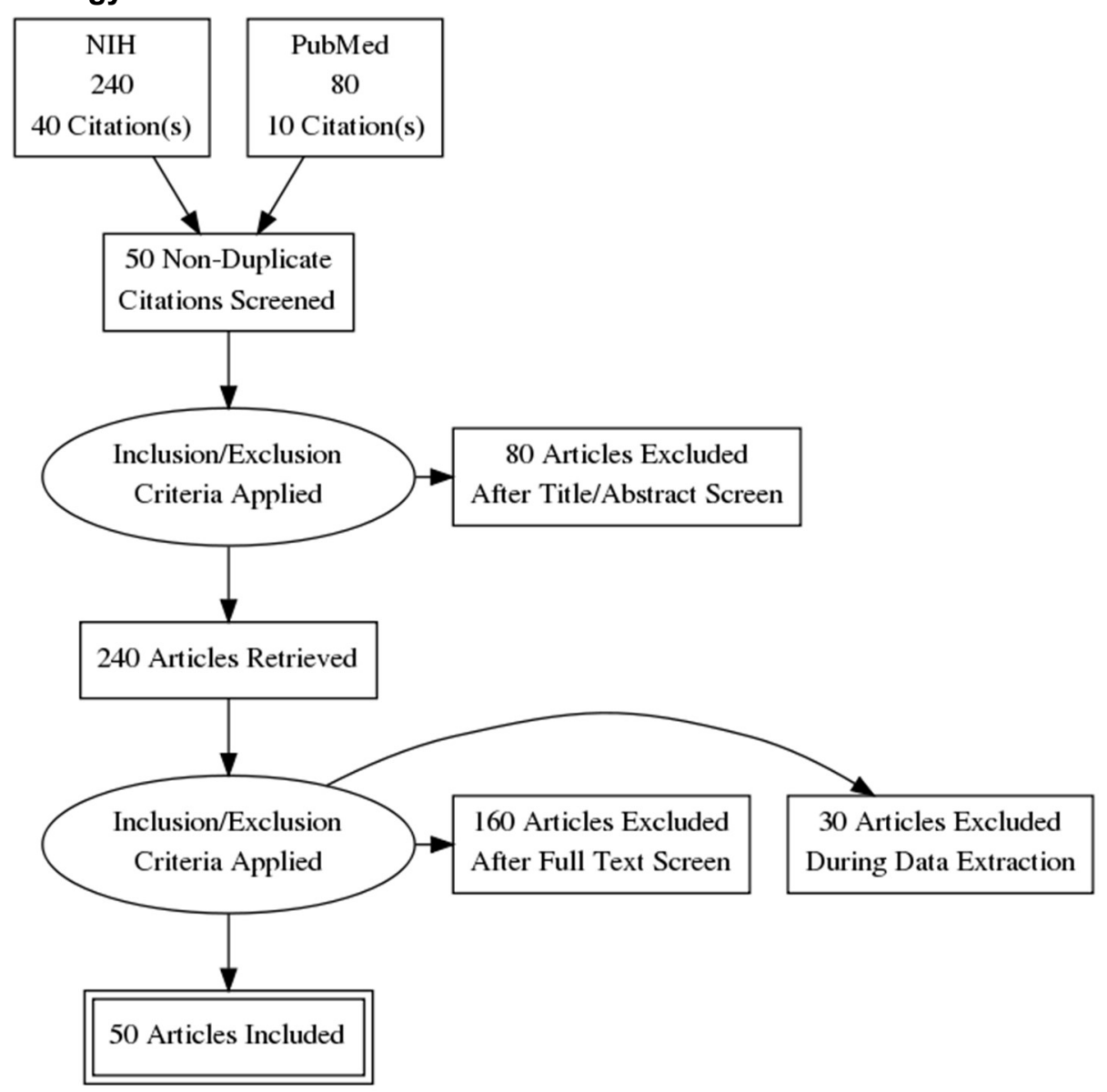

\section{Structure and Mechanism of CCR5 Binding Capacity}


CCR5 is expressed on the CD4 cell surface where CXCR4 and CCR5 expressions are controlled differently during the activation of T cells and with the binding of interleukin type-2 (IL-2). IL-2 can upregulate CCR1 and CCR2 in CD45R01 T cells and increase the T lymphocyte chemotaxis toward CCchemokines [7]. CD4+ T cells are either apart of the T helper 1 or Th1 lymphocytes that release interferon gamma (IFNY) and lymphotoxin that provided cellular immunity to internal pathogens and $\mathrm{T}$ helper cells type 2 (Th2), which secrete interleukins 4 and 5 (IL-4 and IL-5). IL-4 and IL-5 cause an allergic and humoral immune response to parasites. Th2 lymphocytes use CCR3 chemokine receptors. CCR5 and CXCR3 chemokine receptors are specific for CD4+ Th1 and Th2 lymphocytes [50]. The side chains in the CCR5 helical structure contain non-polar residues such as Leucine, Isoleucine, and Valine. The CCR5 structure also consists of aromatic ring residues such as Phenylalanine, Tyrosine, Tryptophan where these aromatic type amino acids are clustered into helices of transmembranes or TM. There are five categories of these types of clusters [9].

The first cluster (AR1)) has Tyr-37, Phe-79, Trp-86, and Tyr-108 each located in TM1, TM2, and TM3, respectively. The second cluster (AR2) contains Phe-85 and Tyr-89, and Trp-94 and Phe-96. Phe85 and Tyr-89 are found in TM2. Trp-94 with Phe-96 is in ECL1. ECL1 is folded where it regulates the orientation of the central part of ECL2. The third cluster (AR3) incorporates TM3, TM6, and TM7 where each can be segmented into an upper layer that is exposed to solvents. TM3, TM6, and TM7 of the upper level involves Tyr-108, Phe-112, and Phe-109 with Phe-113 in TM3. A tyr-251 is located in TM6. The lower level is anchored into side chains of the receptor that contain Tyr-244 and Trp-248 in TM6 with His-289 in TM7. The AR3 cluster maintains the structure of the receptor that also includes Tyr-244 and Trp-248 in TM6 with His-289 in TM7. The AR3 cluster could possibly act as a middle hinge to mediate transmission of signals. The fourth cluster or AR4 contains Phe-166 and Phe-182 in ECL2, Phe-189 and Trp-190in TM5-ECL2, and Phe-193 in TM5. AR4 mediates the correct folding of ECL2. AR5 has four aromatic side chain residues such as Tyr-187 in ECL2 andPhe-260, Phe-263, and Phe-264 in TM6-ECL3. AR5 has increased CCR5 specificity.

Polymorphisms in the CCR5 promoter is linked to the disease progression of HIV. No $C C R 5 \Delta 32 / \Delta 32$ mutations were found in HIV positive individuals where CCR $5 \Delta 32 / \Delta 32$ confers resistance to HIV infection. Specific SNPs of the CCR5 promoters may control HIV progression into AIDS by regulating CD4 T cell counts [10]. CCR5 is the main co-receptor that allows HIV viral entry. The HIV-1 virus uses its Env glycoprotein to bind to CCR5. Viral entry through CCR5 is more common in active HIV replication and in patients with a high HIV viral load. The interaction between HIV Env and CCR5 is needed for inducing apoptosis. The level of CCR5 cell surface expression is essential for cell apoptosis of adjacent cells where increased expression of CCR5 is related to higher frequency of bystander apoptosis. Amplified promoter activity of CCR5 causes higher expression of CCR5 that proliferates apoptosis of CD4 T-cells. The levels of CCR5 alter other HIV pathogenic regulating factors. There is an established relation between high viral load and activation of T cells by HIV infections. Increased viral replication in CCR5 wildtype patients may cause higher levels of immune cell activation.

The 32 base pair deletion of CCR5 can induce the resistance of infection by HIV-1 and limit the progression of the disease. An allele called the $2459 \mathrm{G}$ allele versus the $2459 \mathrm{~A}$ allele of CCR 5 can delay the development of HIV into AIDS. The $32 \mathrm{bp}$ mutation and the $2459 \mathrm{G}$ allelic mutation lowered the activity at the promoter site of CCR5, reduced CCR5 expression, and lessen HIV replication with less proliferation versus the open reading frame of wild type and $-2459 \mathrm{~A}$ alleles. Shied et al. discovered that persons homozygous for the-2459A/A genotypes expressed more CCR5 on the cellular surfaces of CD4+ T cells [11]. High levels of HIV virus proliferation was related to $G / G, A / A$, and $G / A$ promoter genotypes for CCR5. These polymorphisms lead to a severe HIV pathogenesis as the activity at the promoter sites control CD4 T cell apoptotic cell death and reduction [11]. Another seven polymorphisms include: 1 CCR2 
ORF SNP of $190 \mathrm{G}>\mathrm{A}$, res1799864, and six CCR5 single nucleotide polymorphisms of $2773 \mathrm{~A}>\mathrm{G}$ (rs2856758), -2554G>T (rs2734648), -2132C>T (rs41469351), -2135T>C (rs1799988), -2086A>G (rs1800023), and-1835C>T (rs1800024). These SNPs of CCR2 and CCR5 have been divided into seven different human haplogroups known as $\mathrm{HH}$.

CCR5 expression on T-cell surfaces determine HIVIAIDS susceptibility through altering T-cell activation. T cells are activated through DNA methylation of CCR5 cis-regulatory regions or cis-regions. In CCR5 cis-regions for DNA methylation of CpG regions can lower or increase methylation of T Cells, producing higer or lower expression levels of CCR5 [12]. For memory T cells lower methylation increases CCR5 levels versus higher methylation that lowers CCR5 levels. Activation of T-cells by HIV is related to cis-region demethylation [12]. The CCR5 haplotype polymorphisms of CpG-41 is dominant in southern Africa with causing lowered susceptibility to HIV and prolongs the progression into AIDS. Indivdiuals who lack CCR $5 \Delta 32 / \Delta 32$ mutations, but who have hypermethylation cis-regions of CCR5 have genotypes similar to CCR5- $\triangle 32$ heterozygous individuals. HIV-positive patients have highly demethylated cis-regions even with restored CD4+ T-cell counts after antiretroviral treatment [12]. Many single nucleotide polymorphisms have been detected in the promoter regions of CCR5 that are related to HIV into AIDS progression. The SNPs affect 8 promoters of the CCR5 haplotypes such as HHA and HHG that affect CCR5 expression of immune cell surfaces [10]. SNPs that affect CCR5 promoters and lower CCR5 cell surface levels prolong HIV into AIDS disease progression [10]. Neutralizing antibodies were observed to alter diversity of the HIV-V3 codon 18 with a GPGR "crown motif" that has a neutralizing antibody epitope. The different counts of CD4 T cells proves X4 viruses are more pathogenic during HIV transmission, however, this is possible only in CCR5 wt/wt wild type hosts. R5 variants emerge with X4 in hosts with homozygous wildtype expressions of CCR5, but this is not found in CCR5 $\Delta 32 / \Delta 32$ individuals [13]. In normal and healthier immune functions and responses, R5 viruses are more dominant than X4 specific viruses.

To restrict HIV entrance into host cells, CCR5 has been blocked with small molecule inhibitors or antagonists. Maraviroc is one such antagonist that inhibited Leukocidin ED (LukED) destruction of CCR5 ${ }^{+}$ cells similar to blocking HIV infection. LukE can not initiate CCR5 signaling where surface plasmon resonance (SPR) stagnates native CCR5. Studies of LukE and LukD subunits determined that LukE and not LukD forms bonds with CCR5 at specific times with increased saturation at a $K_{d}$ of 39.6/-0.4nM [14]. A human CCR5 structure was built, purified, and then crystallized with Maraviroc bound. CCR5 structures are similar to the CXCR4 receptor. CCR5 has a large loop known as ECL2 which produces a $\beta$-hairpin. CCR5 has an N-terminal region of specific conformations, and ECLs restricted by two disulfide bonds. The disulfide bonds link Cys101 with Cys178 from the ECL2. The second disulfide bond connects a Cys20 from the N-terminus to the Cys269. The CCR5 bonded to Maraviroc complex showed a ligand attached to the bottom of the CCR5 pocket. A nitrogen from the tropane residue group becomes protonated and forms a salt-bridge with the Glu283. The carbon chain length between the two nitrogens of tropane and Glu283 is crucial for anti-HIV infection of the inhibitors [15]. The inhibitors can bind between Glu283 and Tyr251 [15]. Maraviroc bonded inside CCR5 is deeper where it consumes a larger region of the pocket and maintains less contact with the ECLs. The CCR5 binding pocket is more vulnerable and open to inhibitors than the CXCR4 ligand binding pocket.

Maraviroc is known as an inverse agonist for CCR5 where the maraviroc fortifies the CCR5 in an inactive state and form. Many biochemical studies argue that maraviroc and other small-molecule inhibitors have an allosteric interaction with CCR5. The N-terminus of CCR5 with ECL2 determines the binding capacity of chemokine ligands. In a "two-site" model the N-terminus region is a recognition site for chemokines that interact closer to the chemokine core called site 1 . Site 2 incorporates the active site and interacts with $\mathrm{N}$-terminus of the chemokine. Maraviroc delves deep into a cavity in the seven 
transmembrane domain that is directly distinct from site 1, controlling chemokine recognition [15].This deep buried cavity for maraviroc ovelaps site 2. The CCR5 to Maraviroc complex structure shows that Maraviroc blocks chemokine function by inhibiting the activation of the CCR5 receptor through maraviroc interaction with site 2. Maraviroc blocks the allosteric interaction between chemokine ligands and CCR5. The N-terminus of CCR5 and ECL2 influence the binding between gp120 and HIV-1 viral infection. Maraviroc, thus, inhibits the binding of gp120 with CCR5 in an allosteric type of interaction [15].

The third region called the V3 loop of HIV gp120 builds a $\beta$-hairpin structure that determines the cellular types of tropism and co-receptor specific signaling responses. The outer region of the V3 loop causes gp120 to bind to the N-terminus of CCR5 as the V3 crown binds to co-receptor ECL2 with side chain residues in the binding sites used for ligands. $\mathrm{V} 3$ regions of $\mathrm{X} 4$-tropic viruses have more positive charged residues than R5-tropic viruses. The acidic residues in CXCR4 such as Asp97, Asp171, Asp187(ECL2), Asp193, and Asp262 provide critical sites for binding ligands in X4-tropic viruses, which are also pertinent for HIV-1 infection. For CCR5 the acidic side chains are replaced with uncharged residues such as Ser179, Asn258, Gln188,Tyr89, and Gly163. The N-terminus of CXCR4 consists of nine acidic residues, however, CCR5 has three acidic residues where mutations occur to change CCR5 residues into Trp86, Trp248, Tyr108, Trp94, and Tyr251, which this further confirms the significance for the net charge on the V3 loop for the selectivity of the CCR5 co-receptor. These residues can construct a cluster in the CCR5 binding pocket for ligands, forming an auspicious site for gp120 to bind.

The viral envelope glycoproteins found in the $\mathrm{V} 3$ third loop region for gp120 determines the tropic specificity of phenotypes. Mutations and changes in the V3 loops and in the binding pockets for antibodies in this V3 region can interrupt viral entrance via the CD4 T cell binding process [16]. G-proteincoupled seven-transmembrane-segment receptor, known as HUMSTSR, LCR-1, or LESTR Antibodies binding to HUMSTER inhibits the cell fusion of HIV- 1 for its viral infection [Choe]. These $\beta$-chemokines allow the in vitro resistance of HIV-1 infection of healthy individuals who engaged in risky sexual exposures with $\mathrm{HI}-1$ positive partners [16]. Seven transmembrane receptors used for signaling through heterotrimeric $G$ proteins of leukocytes are stimulated by chemokines. Chemokines are a family of peptides that assist with guiding and recruiting leukocytes to sites of inflammation to allow leukocytes to release granules from granulocytes, control avid release of integrins, and produce many proinflammatory responses. The alpha chemokines control monocytes, basophils, eosinophils, and lymphocytes. In the CC chemokine receptor group and family reside CCR1, CCR2, and CCR3, CCR4, and CCR5.

There is a possibility to reengineer cells with CCR5 modifications to render these cells resistant to HIV-1 infection and many other viral infections. These CCR5 edited cells can be transplanted without inclusion of the $2459 \mathrm{G}>\mathrm{A}$ SNP of CCR5. However, the 2459G>SNP of CCR5 can become useful for developing antibodies against CCR5, producing HIV-2 neutralizing antibodies, and for designing HIV-1 therapeutic vaccines [11]. CCR5 antagonists with consideration of $2459 \mathrm{G}>\mathrm{A}$ can be re-examined. Knowledge of whether an individual receiving an immunologic or a chemotherapeutic, has $G / G, G / A$, or an $A / A$ genotype can allow a better prediction of the possible responses to these therapies through a mechanistic type of explanation and modeling.

\section{Frequency of ccr5 SNP Mutations}

To analyze and assess the percentage CD4+CCR5+T cells and the density of CCR5, flow cytometry was used to measure the distribution of CCR5 in 245 individuals [17]. Genetic analysis showed seventy single nucleotide polymorphisms or SNPs, four inserts, and 65 individuals carried the 32-base pair deletion of CCR5. Only Caucasian groups showed the $\Delta 32$ mutation while one individual was homozygous and without CCR5 expression. Eight open reading frame shift mutations were exhibited 
including 12 novel mutations in two ORFs. More genetic variability and diversity of CCR5 was found in the South African black groups where 39 mutations were present [17]. In Sub-Saharan Africa, HIV is the most predominant and prevalent infectious disease. HIV enters into host T cells of the immune system through using its glycoprotein 120 receptor or gp120. The gp120 binds to the host cell receptor called the cluster differentiation four of the CD4+ on the surface of T-lymphocytes. Through membrane fusion the HIV virus can enter into the T cells.

Membrane fusion is completed through the glycoprotein 41 receptor (gp 41). The gp41 activates and initiates the fusion of the virus with the CCR5 or via the chemokine receptor type four (CXCR4). Through the gp41 interaction with CCR5 or CXCR4, the virus is anchored into the host membrane of the T cell. CCR5 is significant for HIV infection where a mutation that deletes 32 base pairs in the ORF of CCR5, provides HIV resistance for homozygous individuals. In heterozygotes the progression of HIV into AIDS is hampered. T cells become resistant to HIV after the 32 base pair deletion in CCR5 denatures its protein, and CCR5 cannot become inserted into the host cell membrane. The virus is unable to fuse with the host cell surface and membrane where it cannot enter the host cells.

After Hutter et al. transplanted stem cells with the $\Delta 32$ mutation, in an HIV-positive individual, the individual was free of viral infection that he maintained for 20 months. Hutter et al., used stem cells from an histocompatibility leukocyte antigen-matched (HLA) HIV-negative donor who was homozygous for the 32-bp CCR5 deletion and mutation. Since 2011, this individual has remained HIV-negative and his immune system has been restored like an $\Delta 32$ homozygous donor. The $\Delta 32$ mutation is rare in the black African population. Many African groups have mutations in the C101X, which also offers HIV resistance. In South Africa, many novel mutations were found that lessens the attachment of HIV to host cell membranes [17]. These novel mutations include D2 V and R225X. The D2 V mutation lessens the binding of HIV to the cell surface of its host. R225X is similar to the $\Delta 32$ mutation where it develops into the ablation of the $\mathrm{R} 225 \mathrm{X}$ receptor expression, eliminating the bond between HIV and the receptor.

In South Africa, studies are being conducted to find novel mutations and genetic variability of CCR5. Many studies emphasize finding novel mutations in exon 3 of the CCR5 gene. Picton et al in 2010 examined the 9.2-kb region of CCR5 from 35 black and white South Africans to discover single nucleotide polymorphisms (SNPs), indels (inserts and deletions), and other haplotypes. Picton et al. found 68 SNPs, five indels, and seven haplotypes. However, studies have not reported how these mutations affected CCR5 expression, individual disease progression, and HIV infection [17]. For this reason, Barmania et al., conducted a study using novel methods that determined the genetic variations in the CCR5 gene and ORF within the South African groups and populations. Barmania et al. chose individuals with low expression of CCR5 via flow cytometry procedures to find novel mutations of the CCR5 gene and to elucidate its phenotype.

The expression of CCR5 on the cell surface of CD4+ T-cells has variations between individuals and populations. Platt et al. characterized 10,000 CCR5 molecules for every CD4+ cell, which is necessary for HIV to infect CD4+ T-cells. CCR5 expressed on the cell surface at levels below the 10,000 threshold negatively affected the HIV virus' ability to infect host cells [17]. However, a few other specific viral types and isolates can use lower levels of CCR5 more sufficiently. CCR5 can alter HIV viral production and the viral load where individuals with a reduced viral load also express densities of CCR5 at levels below the threshold value of 10,000 .

Eleven White South Africans were homozygous for the $\Delta 32$ mutation and had no expression of CCR5 on the cell surface that was measured through flow cytometry. The black South African population lacked the $\Delta 32$ mutation. The mutation of $\mathrm{C} 101 \mathrm{X}$ is a change at a nucleotide of position 303 mainly found 
in the Caucasian population [17]. The C101X mutation has been in the South African population as an heterozygous state only expressed in Caucasions. This mutation originated from a male homozygous person who remained HIV-negative after constant exposure of the virus from a significant other for 10 years [17]. The C101X mutation has less expression of C101X on the cell surface, which provides HIV resistance for individuals. This mutation breaks disulfide bonds of cysteine residues that terminates the translation of the $\mathrm{C} 101 \mathrm{X}$ receptor.

A mutation exclusive for the black and colored South African groups and populations is a mutation at nucleotides of the 673 position named as R225X. R22X mutations were discovered in two black Africans that expressed this mutation at a heterozygous state [17]. The mutation of R22X is found at the third intracellular loop (ICL)and replaces an arginine with a stop codon that prematurely stops expression of the R225X mutation. Folefoc et al. formed a mutant receptor with this R22X mutation, and the expression of CCR5 was absent [17]. Inhibiting R22X, in this manner, lessened HIV attachment to cells, and these cells were resistant to HIV infection through reduced interaction with CCR5. The homozygous state of the R225X mutation may offer HIV resistance [17].

In the intron of region one for the CCR5 gene position at -2273, a novel SNP was recognized and identified. The A to G SNP mutation was found in two South African blacks [17]. The intron 1 locus is significant for controlling CCR5 expression where it constructs the programmed death PD promoter. This region of intron 1 consists of cis-acting compounds that alter CCR5 expression. The mutation at intron 1 of CCR5 affects the start codon known as ATG. Mummidi et al., confirmed these ATG start codons located upstream regulate CCR5 expression where minicistrons upstream monitor the translation of CCR5 [17]. Another novel mutation is located in the ORF of the CCR5 gene at the nucleotide position of 667. This mutation is a C to T SNP identified in two blacks who were heterozygous, and two Caucasions in South Africa. The mutation at the 667 position of CCR5 affects the third ICL in the CCR5 protein and substitutes an arginine as a non-polar tryptophan. This arginine residue is completely conserved. A R223Q mutation is located in this 667 position and changes the function of HIV to co-receptor interactions while the co-receptor still binds to gp120. A novel mutation known as R223W that has a similar effect as the R223Q mutation.

At position 817 there was a mutation detected in one black South African who was heterozygous for this mutation. This individual expressed the R223W mutation as well. The mutation at position 817 is an A to C SNP that changes a polar asparagine into a basic and alkaline histidine residue (N273H). $\mathrm{N} 273 \mathrm{H}$ mutations are on the third Extracellular loop (ECL), but its effects need further study. The 5'untranslated region UTR, introns, and 3'UTR regulate CCR5 gene expression. The point mutation of $C$ to $\mathrm{G}$ in the SNP at position 10 was identified in heterozygous black South Africans is significant because it initiates translation.

About 75 mutations of CCR 5 have been found [Barmania]. These mutations include 63 previously recognized and 12 novel mutations. Seventy of these 75 mutations were SNPs, four point mutation inserts, and one $\Delta 32$ mutation. The black South Africans displayed 39 mutations, and 13 of the mutations belonged to Caucasian South Africans [17]. The 3' flanking region and intron 2 exhibited the maximum number of mutations. There were 12 mutations only detected in the population of South Africa. The ORF of CCR5 consisted of nine of these previously known 12 mutations. Blacks of South Africa studied in their research retained five of these 12 mutations, and four were recognized from the Caucasian groups. The ORF displayed zero mutations in each two racial groups of South Africa. The $\Delta 32$ mutation was located in 12 Caucasian people and none were identified in South African blacks. Eleven individuals were heterozygous for the $\Delta 32$ mutation and only one Caucasian was a homozygous male [17]. 
A total of 12 novel SNPs were detected in the Barmania et al. study, which consisted of two SNPs in the ORF of CCR5. Seven mutations were found from Black South African groups, and three Caucasians had exclusivity for three of these twelve mutations [17]. More research is needed to analyze novel mutations of the CCR5 receptor focused specifically on the binding of ligands and HIV to CCR5 coreceptor interactions [17]. Flow cytometry can reveal the indicators of CCR5 cell surface expression may not result from a single mutation. Because Barmania et al. identified low numbers of CCR5 expressers, they could detect more novel mutations of the CCR5 gene. More black South Africans expressed these novel CCR5 mutations, showing more genetic diversity where 39 of these mutations were in black South African groups.

\section{Types of CCR5 SNPs}

In Rwanda, 1661 people and 1649 individuals carried two wild-type alleles for CCR5 [18]. Twelve individuals carried a heterozygous genotype of hCCR5D24. Two of the 83 individuals were heterozygous for the CCR5D24 genotype versus 6 of the 613 and two of the 579 HIV-1 positive individuals also showed this heterozygous allele of hCCR5D24 [18]. Many LTS and ESN individuals showed the highest rate and prevalence of hCCR5D24 mutations. None of the participants had a homozygous genotype for hCCR5D24. The heterozygosity of the hCCR5D2 mutation in LTS and ESN groups exhibited a delayed progression of HIV-1 and provided protection from the transmission of HIV. The hCCR5D24 deletion was detected in other populations of Africans [18]. The hCCR5D24 deletion was not found in any of the 224 infants with HIV-1 positive mothers [18]. None of the 500 HIV-1 negative and 200 HIV-1 seropositive Causcasions showed the deletion [18]. This notes the specificity of the hCCR5D24 mutation mainly localized in East Africa. The frequency of the CCR5D32 deletion is widely distributed in Caucasions but with lowered expression in Africans and Asians. The CCR5D32 allele is more frequently distributed in Europe because of the black plague and smallpox placing positive pressure on selecting the CCR5D32 allelic mutation [18]. The hCCR5D32 mutation may increase the susceptibility of tick-borne encephalitis and from the West Nile virus causing infection. Likewise, the hCCR5D24 deletion in Africans may have been chosen to provide immunity against other infectious diseases [18]. Individuals homozygous with a wild-type of CCR5 more frequently distributed in the Crimena-Congo in patients who were infected with haemorrhagic fever. In CCR5 knock-out mice, the mice were resistant to lethal infection by the Dengue virus. The discovery of hCCR5D24 was incredible.

Many more research needs to elucidate the prevalence of the hCCR5D24 deletion in East African individuals versus its absence in Guinea Conakry or West Africa such as Luxembourg [18]. The absence of the deletion in West Africa may be attributed to a positive selective pressure caused by an increased or decreased infection susceptibility. The African population has a diverse ancestral genetic history shown in genetic clusters that match the many different ethnicities only grouped by shared cultural and language dialects. This diversity could describe and explain the reason for the hCCRD24 widely distributed in East Africa. The hCCR5D24 deletion may prolong the development from HIV-1 to AIDS. However, the detection for the heterozygosity of hCCR5D24 had a limited size of participants, the reduced frequency of hCCR5D24 alleles, and the lack of homozygous participants with hCCR5D24 did not yield strong statistical results and evidence [18]. Individuals homozygous for hCCR5D24 were protected from HIV-1 infection. The hCCR5D24 deletion caused the CCR5 coreceptor to be truncated that eliminated its cell surface expression like other natural mutations of CCR5. HEK-293T and HeLa-CD4 cells were transfected with a pCMV5 plasmid consisting of a wild-type hCCR5 or with sequences of hCCR5D24 that added $\mathrm{HA}$ tags to the $\mathrm{N}$-terminus of the co-receptor.

The mRNA of wtCCR5 and hCCR5D24 were discovered to be expressed in each cell after 24 hours after the transfection. Confocal microscopy of the receptor cell surface and the expression of CCR5 
was examined. The HA tagging and stain exposed the hCCR5D24 receptor was not expressed at the surface of the cells, but that is aggregated in the cytoplasm of the HEK293T and HELa-CD4 cells. The CCR5 was formed but it remained intracellular inside the cytoplasm. Flow cytometry further determined the lack of CCR5 expression in the mutants. CCR5 was stained and its expression screened in GFPpositive cells chosen for transfection. The GFP-negative cells did not express wt or mutant CCR5 deletion. The hCCR5D24 deletion was undetectable in ECL2 targets. This suggests that hCCR5D24 deletion was localized above at the TM2 adjacent to disulphide bridges connecting ECL1 to ECL2, which caused an alteraction in ECL2 conformation. The change in conformation can result in misfolding of proteins that may interrupt its expression at the surface with its reduced export to the cell surface. The hCCR5D24 mutation was confirmed to only be expressed intracellularly after performing micrographs with T21/8 antibodies. Anti-HA antibody tags displayed absence of the mutant CCR5 protein at the surface.

HeLa-CD4 cells were examined after transfection with HA-hCCRD24 or FLAG-wtCCR5 or cotransfected with FLAG-wtCCR5-FLAG and HA-hCCRD24 to investigate the effect of hCCRD24 on HIV-1 infection. After 48 hours post-transfection, the cells were infected with CCR5 or CXCR4 specific HIV-1 pseudotype viruses [18]. The HeLA-CD4 cells that expressed CXCR4 without CCR5 were injected with HXB2 and without R5 tropic pseudotyped viruses. The HeLa-CD4 cells with CCR5 expressed on its cell surface exhibited increased R5 tropic HIV-1 infection. The HeLa-CD4 cells with hCCRD24 mutations showed lack of bronchoalveolar lavage pulmonary infections (BaL) and HIV-1-ADA8 infectious particles. HeLa-CD4 that were cotransfected with wtCCR5 and the hCCRD24 mutation controlled the entry of ADA8 and BaL virions similar to the HeLa-CD4 wildtype of CCR5 cells [18].

de Oliveira results confirm the previous research studies of the malfunctioning or lowered expression of CCR5 at the surface for many mutants of CCR5 proteins such as the CCR5-delta 32 base pair deletion, human CCR5 FS299, C101X, and CCR5-893 mutations. Another mutation known as the A73V mutant is localized in the transmembrane region near the domain near the 24-base pair deletion of CCR5 [19]. Lower levels of CCR5 were previously described in other homozygous hCCR5D32 individuals. The protozoan known as Trypanosoma cruzi causes the Chagas disease in Latin America. Because of the migration of people from Latin America to other countries, the Chagas disease has spread. The World Health Organization approximates that 7 million people were infected with $T$. cruzi. The symptoms of the disease occur after two decades post-infection where $30 \%$ of individuals with the infection display chronic Chagas heart disease (CCHD) [19].

Genetic mutants of the cytokines affect the pathology of CCHD. Chemokines are cytokines produced by small proteins to aggregate and recruit leukocytes to sites of inflammation. The chemokines bind to G-coupled receptors that consist of 7 transmembrane domains. Many types of chemokine receptors exist in leukocytes. The CCR5 has ligands known as $\mathrm{CCl} 3, \mathrm{CCL} 5$, and $\mathrm{CCl} 3$ that are expressed in monocytes, macrophages, and in T cells. Individuals who expressed more of the CCR5 protein had a higher rate of cardiomyopathy. A reduced cardiac function was related to lowered expression of CCR5. CCR5 gene polymorphisms were related to CCHD. Antibodies against anti- $T$. cruzi was detected by ELISA. PCR identified the polymorphisms of CCR5 32 and CCR5 59029 A/G. A chi-square analysis tested the variables between each of the two polymorphisms and groups. The AA genotype showed a higher frequency in CCHD patients versus the control group [19]. The CCR5 32 polymorphism did not cause the symptoms of the CCHD to occur. However, the CCR5 59029 A/G polymorphism led to varied forms of CCHD susceptibility [19]. 


\section{Effects of CCR5 SNPs on Infection and Disease Part 1}

Hepatitis B viruses infect the human liver. Many researchers argue that variations in genetic and immunological patterns for patients with HBV may cause the infection. In previous studies researchers determined that chemokines regulate the transport and activation of immune cells in response to HBV infection. The chemokines responsible include MIP-1a, MIP-1b, and RANTES that bind and act through CCR5 [20]. CCR5 is expressed on many immune cells such as T cells, NK cells, and macrophages. CCR5 monitors the initiation and transport of immune cells when immune cells gather immune responses against HBV and other viruses. Mutations in the expression or function of CCR5 cause weakened immune responses to combat HBV. A 32 base pair deletion in exon 1 of CCR5 with three different polymorphisms in the promoter sites of CCR5 and this downregulated the co-receptor [20]. The expression of CCR5 changed during hepatitis B; however, the role that each of these mutations play in Hepatitis B is still preliminary and uncertain.

The disorder known as Diabetic Nephropathy (DN) leads to high death rates in patients with diabetes mellitus. Previous studies have confirmed the relation between DN and the CCR5 59029G/A polymorphism, but still the results are controversial. Cao et al. used a meta-analysis to study the association between DN and CCR5 59029G/A polymorphism. Cao et al. found the CCR5 59029G/A polymorphism displays a substantial increased risk of developing DN [21]. Publications were reviewed and searched in digital databases and many alternative sources. Cao et al. used pooled odd ratios (ORs) with a 95\% confidence intervals (95\% Cls) to show the intensity between the CCR5 59029G/A mutation and DN prevalence [21]. They determined that the CCR5 59029G/A polymorphism is parallel to increased susceptibility to DN more specifically in Asian populations and groups with type 2 diabetes mellitus.

CCR5 is involved in chemotaxis, the proliferation, and in the immunoregulation of cells that cause inflammation. The polymorphism of CCR5 59029G/A is located in the promoter area of CCR5 genes that can change the level of expression and function of the CCR5 co-receptor, which is related to the individual susceptibility to DN. Ahluwalia et al, discovered a higher rate and frequency of the AA genotype in the CCR59029G/A polymorphism for individuals with DN who live in North and South India. This proves the increased susceptibility of this AA genotype [21]. Caucasians who live in Poland were found to increase the risk of DN development that showed the effect in the A allele also expressed for people with type 2 diabetes. The A allele and AA genotypes were also prevalent in Chinese populations. Mlynarski et al. demonstrated the increased susceptibility of type 1 diabetes for male carriers of the $G$ allele of this mutation and exhibited a 1.9-fold higher prevalence of $D N$ development versus non-carriers of this $G$ allele [21].

CCR5 gene polymorphisms may interact with tumorigenesis. The CCR5 $\Delta 32$ polymorphism may produce higher rates of breast cancer (BC) susceptibility. Li et al. performed a meta-analysis. The $\Delta 32$ polymorphism of CCR5 can reshape proteins abnormally that may act in the pathogenesis and frequency of many different types of cancers such as BC [22]. A Turkish population was studied and the heterozygous genotype of the $\triangle 32$ CCR5 polymorphism was found to be associated with the increased risk and indicators of breast cancer pathogenesis [22]. The chemokine, CCL5 that binds to its receptor of CCR5 can contain polymorphisms associated with coronary artery disease (CAD) in Taiwanese populations but these mutations have not been researched or deeply analyzed. In a study by Ting et al. 483 participants were examined [23]. These individuals received a coronary angiography while admitted into Chung Shan Medical University Hospital. The polymorphisms known as CCL5-403 and CCR5-59029 were identified through polymerase chain reaction-restriction fragment polymorphism methods. They determined that CCL5-403 combined with a TT genotype lead to an increased risk of CAD development. The occurrences of CCR5-59029 with GG or GA genotypes were more frequent than the AA genotype in 
individuals with acute coronary syndrome. CCL5-403 or CCR5-59029 point mutation polymorphisms may contribute to and predict a cardiovascular event [23].

For chronic renal failure (CRF) individuals and treated with hemodialysis, there is genetic cause from the CRF association with fractalkine receptors such as CX3CR1, T280M, V2491, and CCR5-59029 $\mathrm{A} / \mathrm{G}$ gene polymorphisms. Researchers sought to confirm the links between these gene polymorphisms with CRF, hypertension (HT), diabetes mellitus (DM), and atherosclerosis (AS). Approximately $225 \mathrm{CRF}$ individuals who underwent hemodialysis and 201 controls were recruited into this study. CRF individuals were subdivided into three categories according to their chronic conditions $H T=127, D M=65$, and $A S=33$ [24]. Their genotypes were determined through the polymerase chain reaction-restriction fragment length polymorphism procedures. The genotypic allele frequencies of CX3CR1 V249I polymorphism occurred more in CRF, CRF and DM, and CRF with AS subjects versus the healthy controls at $p<0.05$. The frequency of the $G$ allele in the CCR5 gene polymorphism was more prevalent in CRF with DM patients than the controls. The GG genotype and $G$ allele frequencies in CCR5 polymorphisms were higher in CRF with AS subjects versus their controls at $p<0.05$ [24]. There was an immense relation between CRF individuals with $\mathrm{HT}$ and without $\mathrm{HT}$ in the genotypic and allelic frequencies of the $\mathrm{V} 249 \mathrm{l}$ polymorphisms $(p<0.05)$ [24]. For the CX3CR1 T280M polymorphism was not different in any of the comparisons of genotypes and alleles. There are significant associations between CX3CR1 V249I and CCR5-59029 A/G polymorphisms with $\mathrm{HT}, \mathrm{CRF}$, and AS individuals.

To better understand the connection between genetic polymorphisms of CCR5 and diabetic nephropathy (DN). The polymorphisms include the monocyte chemoattractant protein-1 (CCL2rs3917887), chemokine receptor 5 (CCR5-rs1799987), engulfment and cell mortality (ELMO1-rs74130), and interleukin-8 (IL8-rs4073). These SNPs can develop into DN for people with type 2 diabetes (T2DM). Yahya et al. examined 1,000 diabetics in Malaysia where 652 T2DM patients were tested [25]. Procedures were performed using the DNA Sequenom mass ARRAY to detect CCL2, CCR5, IL8, and the ELMO1 polymorphisms. DNA extractions were taken from T2DM Asian Individuals. The genotypes and alleles were analyzed through genetic models. For the CCR5 rs1799987 polymorphism the $\mathrm{G}$ allele occurred more frequently than A. The higher allelic frequency of G for CCR5 rs1799987 was associated with a higher prevalence of diabetic nephropathy in the Chinese populations [25]. The higher allelic frequency of A over T was associated with more cases of nephropathy in Indian groups. The gene pool of these genetic variations are different for each ethnic group and population. More studies are needed that focus on the environmental influences for these variations in CCR5 rs1799987 genotypic and allelic frequencies in diabetes nephropathy [25].

Song et al. sought to determine whether the (CCR5- $\Delta 32)$ polymorphism is associated with type 1 diabetes, asthma, and Bechet's disease (BD). A meta-analysis of 9,656 cases revealed a negative interaction between T1D and CCR5- $\triangle 32$ [26]. There was a positive relation between BD and the $\Delta 32 \Delta 32+\Delta 32 \mathrm{~W}$ genotype. No associating was discovered between CCR5- $\Delta 32$ and asthma. The metaanalysis showed the CCR5- $\triangle 32$ polymorphism is a preventive factor for T1D in Europeans, but a disease causing factor in BD HLA-B51 carriers [26].

Slominski et al. aimed to analyze the association between CCR5- $\Delta 32$ polymorphism and the susceptibility of diabetic retinopathy in patients with type 1 diabetes. They examined 420 patients with 350 healthy controls for the CCR5- $\Delta 32$ polymorphism by observing CRP and TNF-alevels in the serum [27]. The adhesion molecules known as VCAM, ICAM-1, and ICAM-3 were examined. The CCR5 ligand of MCP-1 was measured. There was a negative relation between DM1 and the $\Delta 32$ allele. The $\Delta 32$ allele occurred at a higher frequency in the DR group versus the control. The $\Delta 32$ carriers produced the highest 
levels of adhesion molecules, markers of inflammation, and the CCR5 ligand. Their findings confirm that the CCR5- $\Delta 32$ polymorphism is related to DM1 where the $\Delta 32$ allele prevents and protects against developing diabetes type 1 , but it amplifies the risk of developing DR patients who already have the disease [27 2017].

Researchers examined the role the CCR5- $\triangle 32$ plays in the development of dyslipidemia in children who are type 1 diabetics at risk for suffering from cardiovascular disease. Close to 380 patients who have T1D were screened to characterize the CCR5- $\Delta 32$ genotypes examined through the polymerase chain reaction [28-Słomiński 2019]. After screening the participants, $\Delta 32$ carriers had higher levels of $\mathrm{HbA} 1 \mathrm{c}$, blood glucose, LDLs, and lower levels of HDI than noncarriers. The minor $\Delta 32$ allele showed a higher frequency of the dyslipidemic patients than the healthy controls. An increase in the risk factors for dyslipidemia was higher as well. The CCR5- $\triangle 32$ polymorphism is related to higher plasma lipid values and the $\Delta 32$ allele increases the perceptibility of developing dyslipidemia in patients with T1D [28Słomiński, 2019]. Recognizing and Identifying the variants of the $\Delta 32$ allele may guide the discovery of a novel method for treating dyslipidemia in T1D patients.

\section{Effects of CCR5 SNPs on Infection and Disease Part 2}

For HIV-1 to gain entry into CD4 ${ }^{+} \mathrm{T}$ cells it must bind to CD4 via interaction with CCR5 or CXCR4 coreceptors. A drug or genetic disruption of these coreceptors protects cells from HIV-1 infection or other viruses that use CCR5 and CXCR4 for infection. Didigu et al. used zinc-finger nucleases to genetically modify ccr5 and cxcr4 in human $\mathrm{CD} 4{ }^{+} \mathrm{T}$ cells. The modified $\mathrm{T}$ cells proliferated and showed ample resistance to CCR5 and CXCR4 HIV-1 entry in vitro. After transplanting the modified cells into humanized mouse models for HIV-1 infection, the mice showed protection from HIV-1. Disrupting CCR5 and CXCR4, may offer an advantageous method for providing long-term and drug-free treatment of HIV-1 infections [29].

In 2007, Charite Berlin and his team transplanted stem cells in HIV-positive individuals with acute myeloid leukaemia (AML) using cells with the CCR5-delta32 deletion. After 3 years of his initial treatment, the patient remains free from taking antiretroviral medicines and HIV-negative [30]. The patient's CD4 cell count increased by more than $800 / \mu l$ with all stem cell derivatives and gut macrophages became negative for CCR5 expression [30]. This has given much new hope for some type of gene therapy that may improve the treatment of HIV. This single case has caused the repetition of this approach. CCR5 induces movement of cancer cells known as metastasises and increases inflammation. CCR5 improves DNA repair, provides more cell survival, resists DNA damage, and activates invasion of metastasis. CCR5 expressed on CD4 T cells in cancer epithelial cells produces the metastasis of cancer cells. Smallmolecule inhibitors specific for CCR5 can inhibit metastasis of oncogenic breast cancer cells and prevent metastasis from prostate cancer in mice. In vivo CCR5 caused metastasis in p53 specific breast cancer cells. Silencing CCR5 with siRNA did not lessen the phenotype of metastasis termed MDA-MB-231.

CCR5 ligands can cause tumorigenesis and prometastatic inflammation. There is a possible synergy between CCR5 inhibitors and immune checkpoint inhibitors that have been proven in Pfizer and Merck clinical trials [31]. In Pfizer and Merck trials CCR5 inhibitors were found to combine with checkpoint inhibitors that recruited immune cells such as tumor-infiltrating lymphocytes (TIL), MDSCs, tumorassociated macrophages (TAM), natural lymphoid cells, Tregs, dendritic cells, and mesenchymal stem cells [31]. These recruited cells brought about tumor-induced immunosuppression. Through immunosuppression tumors are able to evade immune responses by inducing tolerance of the immune system through this recruitment of CD4pCD25pFoxp3pTregs. 
CCR5 induces angiogenesis and resistance to apoptosis. CCL5 causes effects from angiogenesis by propagating migration of endothelial cells, spreading, VEGF secretion, and formation of neovessels. Tumor cells when stimulating CCL5 it produces VEGF, and the secretion of CCL5 causes the recruitment of TAMS-expressing CCR5. Inhibitors of CCR5 lessen lymphangiogenesis. Cells in tumors need more glucose uptake with CCR5-induced phosphorylation of Akt that stimulates increased uptake of glucose. Fatty acid synthesis and glycolysis increases at a higher rate as well. After analyzing breast cancer cells it showed that CCR5 controls RNA activation for PI3K/Akt, cell survival signalling pathways, and biogenesis of ribosomes.

Endothelial progenitor cells or EPCs are required for forming vascularization in tissues for repair and tumorigenesis. EPCs migrate from the bone marrow (BM) through tissues by the circulation of blood and chemotaxis. When mice were absent from CCR5, the vascular EPC was lessened, which prolonged wound health of the skin. These CCR5 modified cells were transplanted into mice with $\mathrm{Ccr5}^{-/}$mice, $\mathrm{Ccr5}^{+/+} \mathrm{BM}$ cells, but not into $\mathrm{Ccr}^{-/}$cells where these cells gathered at the wound site [32]. At the wound site in $\mathrm{CCr}^{-/}$mice these cells were blended into the vasculature and this restored the innate neovascularization. CCL5 led to in vitro migration of EPC in which it was CCR5-dependent. VEGF and TGF- $\beta$ was significantly lowered at wound sites in most $C c r 5^{-/}$mice; therefore, EPCs are a prominent source of growth factors for tissue repair. CCL5 to CCR5 may become a novel target site for controlling neovascularization for providing tissue and wound repair [32].

Immune responses affect the progression of colorectal cancer (CRC). Two sets of myeloid cells causes an influx of T cells via CXCL9/CXCL10 where CCL5 is produced by the $T$ cells. These $T$ cells stimulate tumor growth effects through CCR5. By blocking CCR5 expression a repolarization of macrophages occurs with anti-tumor effects. These anti-tumor effects were proven in phase I clinical trials, using a CCR5 antagonist in patients who have liver metastases in advanced CRC. Mediation of these tumor responses were observed in CRC. CD4+ and CD8+ T cells promote tumorigenesis in metastatic colorectal cancers. These two $\mathrm{T}$ cells proliferate the production of tumorigenic inflammatory cytokines controlled by CCL5-CCR5 interactions. Tumors are proliferated through migratory characteristics in tumor cells that produce pro-metastatic factors. The pro-metastatic factors are produced by tumor-associated macrophages. When blocking CCR5, CCR5 is a target for activating anti-tumoral repolarization of macrophages [33]. An effective therapy for blocking CCR5 can lower pro-tumor inflammatory microenvironments through directly affecting tumor cells and macrophages involved in tumorigenic production [33] .

Chemokines and their receptors contribute to the disease causation of atherosclerosis and heart diseases. The chemokine receptor known as CCR5 initially was only known by its association with HIV infection of macrophages and T cells. Currently, new evidence shows CCR5 and its ligands such as CCL3, CCL4, and CCL5 (RANTES), affect the activation and development of atherosclerosis [34]. Deleting CCR5 in the CCR5 delta 32 polymorphism, provides resistance to HIV infection, and is now associated with a lowered risk of developing heart disease [34]. Blocking CCL5 can reduce atherosclerotic issues in many animal models. CCR5 with its ligands are recognized in human and mouse vasculature where these ligands have been screened in atherosclerotic plaque. CC chemokines are also connected to saphenous vein graft diseases [34].

Chemokine- receptors with ligand systems in atherogenesis is proposed where CCR5 recruits monocytes. These monocytes develop into plaques. Many HIV-infected individuals develop heart diseases. Therefore, a greater emphasis and focus needs to be placed on drugs that block CCR5 
receptor interaction with its ligands. Maraviroc drugs give CCR5 as a target for preventing cardiovascular disease in HIV-positive individuals [35]. CCR2 and its ligand known as monocyte chemoattractant protein (MCP-1), are crucial for attracting adipose tissue macrophages (ATM) when developing resistance to insulin. Expression levels of CCR5 with its ligands were examined for causing obesity-induced inflammation of adipose tissue and resistance to insulin. The levels of CCR5 with its ligands were analyzed in white adipose tissue (WAT) with ob/ob and high-fat diet related obesity (DIO) of mice. CCR5 was upregulated in WAT in CCR5+ cells within ATMS versus high-diet mice. CCR5 2/2 mice were resistant to induced insulin resistance, protected from glucose intolerance, and liver disease through $\mathrm{HF}$ diets. Loss of CCR5 reduced ATM and shifted ATM polarization. Transplanting CCR5 2/2 into bone marrow protects against glucose intolerance [35]. CCR5 can critically affect insulin resistance [35].

CCL5 and CCR5 receptors during a microarray analysis of 2,254 human breast cancer samples were found with amplified CCL5 expression with its receptor CCR5 without interacting with CCR3 in basal and HER-2 genes [36]. Many human breast cancer cell lines express CCR5 in response to CCL5.

Oncogenesis induced expression of CCR5 with subtypes of immune cells with functional CCR5 receptors. Antagonists of CCR5 such as maraviroc and vicriviroc block CCR5 HIV interactions with the coreceptor lessened the migration of basal breast cancer cells without lowering proliferation of immune cells. Maraviroc lowered metastasis of breast cancer cells into the heart. CCR5 antagonists as an adjunctive therapy can reduce metastasis of basal breast cancers [36].

\section{Effects of CCR5 SNPs on Infection and Disease Part 3}

Myeloid-derived suppressor cells (MDSCs) are characterized as a heterogeneous population of cancerous myeloid cells that inhibit the response of T cells. MDSCs aggregate in many organs and in tumor tissues as tumorigenesis occurs. MDSCs lack in-depth research. It was found that MDSCs produce higher levels of nitrous oxide and arginase- 1 with increased expression of chemokines such as CCR5 ligands [37]. These CCR5 include CCL3, CCL4, and CCL5. MDSCs were extracted from B16 melanomas from skin in tumor-bearing transgenic mice that showed high levels of CCL3, CCL4, and CCL5. T cells expressed many CCR5 receptors on the surface. The MDSCs sequestered many T cells through CCR5 activity in vitro. Injecting CCL4 or CCL5 intratumorally increased the number of regulatory T cells (Tregs) and the absence of CCR5 lowered the amount of Tregs [37]. In mice deficient of CCR5, tumor growth was prolonged, which proves the significance of CCR5 for controlling antitumor immune responses. When MDSCs release chemokines, this accumulates large numbers of Tregs where these MDSCs can be suppressed and used in clinical cancer immunotherapies.

Myocardial infarction induced inflammatory responses that cause cardiac remodeling. To repair the heart after a heart attack relies on mechanisms that regulate and inhibit inflammation to decrease extra damage of heart tissue. Chemokines transport into the infarcted heart that guides the recruitment of leukocytes with many different and distinct characteristics. CCR5 blocks excessive inflammation after a heart attack by aggregating mononuclear cells. The mononuclear cells can suppress inflammation. CCR5 and its ligands such as macrophage inflammatory protein (MIP) $-1 \alpha$ and MIP-1 $\beta$ were recruited and activated by infarcted mice in the myocardium. Forty percent of the mononuclear cells fill and import into the infarcted region and express CCR5 on the surface of the mononuclear cells [38]. Mice without CCR5 expression upregulated proinflammatory cytokines and chemokines in the area of the infarct attack.

The wild-type of infarcts with CCR5 expression on mononuclear cells showed less inflammation with more levels of IL-10 than CCR5 negative cells. Mononuclear cells extracted from CCR5 negative infarcts exhibited less IL-10 expression. This showed evidence of suppression of inflammation. Increased 
inflammation without CCR5 was related to a dysfunctional recruitment of CD4+/foxp3+ T cells [38]. T cells expressing CCR5 showed amplified levels of IL-10 with a potent activity of anti-inflammation. Infarcts negative for CCR5 was due to increased matrix metalloproteinase (MMP) expression, which results in a larger cardiac dilation. CCR5 mediation of T cells aggregating can lower inflammation of a heart attack that ceases damage to the tissues of the heart [38].

The genes of CCR5, MCP-1, and CCR2 have properties that are highly polymorphic. These polymorphisms of chemokine receptors result in rejection of transplants. The polymorphisms of chemokine receptors were analyzed in 173 kidney transplant recipients and 169 healthy control participants [39]. Patients who participated were divided into two groups. Group-1 (G-1) consisted of 33 HLA-identical recipients, and group-2 (G-2) included 140 non-matching transplant recipients. Approximately, 42 patients experienced acute rejection (ARs) with 7 in G-1 and 35 of the 42 in G-2 [39]. Thirteen patients from G-2 had chronic allograft dysfunction (CAD) [39]. The genotypes and the frequency of polymorphisms were studied and the distinction between G-1 patients and G-2 recipitenst was not significant. There was an increased risk of acute kidney transplant rejection discovered in G-1 patients with the CCR2-64I allele. The link between CCR2-64I polymorphisms and CAD was not significant. CCR2-64I was associated with AR in kidney transplant after renal allografts [39].

Sarcoidosis and Crohn's disease have heterogeneous systemic properties in diseases caused by granulomatous inflammation. Caspase recruitment domain (CARD) is a gene that increases the susceptibility of Crohn's disease. The CCR5 gene has been linked to parenchymal pulmonary sarcoidosis and perianal Crohn's disease. In 185 Caucasian patients with sarcoidosis, their genotypes for CARD15 and CCR5 polymorphisms were analyzed [40]. The data was compared against healthy controls to observe associations with pulmonary function tests and radiographs of the chest. It was found that CARD15 genotypes were not different between sarcoidosis patients and the controlled patients with the polymorphism of CCR5 known as $2104 \mathrm{~T}$ ( $702 \mathrm{~W}$ ) had a high pre susceptibility to stage 4 diseases [40]. A carrier of 2104T showed more damage to the lungs while the CARD15 $1761 \mathrm{G}$ polymorphisms increased the improvement of lung function and activity.

Applying therapy such as tumour-infiltrating lymphocytes (TILs) induces complete responses (CR) for $20 \%$ of patients ill with metastatic skin cancer [41]. Aggregating T cells by interaction between CXCR3 or CCR5 with chemokine ligands is pertinent for immune rejection. It was hypothesized that polymorphisms with expression of CXCR3 or CCR5 for TILs medigate the transport of TILs to tumor tissues. TILs were isolated from 142 patients with metastatic melanoma who participated in clinical trials were studied [41]. The patients' genotypes were studied for detection of CXCr3 rs2280964 and CCR5$\triangle 32$ mutation to block CCR5 expression on the cell surface. The CXCR3/CCR5 in TILs, CXCR3/CCR5, and the genes of ligands were screened in 113 tumors [41]. All data of TILs were confirmed via flow cytometry. CR was associated with the CCR5- $\triangle 32$ polymorphism. The lack of CXCR3 and CCR5 expression increased the response to therapy. Upregulation of CXCL9, CXCL10, CXCL11, and CCL5 for pretreated biopsies of tumors was related to an increased overall response (OR). Upregulation of $C X C L 9$, CXCL10, CXCL11, and CCL5 for pretreated tumors caused more response to therapies. The lack of CCR5 in the $\triangle 32$ polymorphism and less CXCR3/CCR5 expression downregulated TIL receptors and controlled the overall response.

\section{Possible CCR5 Targeting Therapies}

Designer nucleases can perform gene editing of targets that are composed as restriction enzymes that can cleave specific DNA sites and targets. After repair of the cuts or breaks, the cell restoring pathway inserts small mutations into the site of the cut or break. This disrupted the DNA target 
site where the function of the protein of CCR5 will be reduced [42]. Vaccines for HBV may become less effective in producing an immune response due to CCR5 polymorphisms [43]. Antagonists used to block CCR5 protein function can prevent and treat HIV and improve the process of organ transplantation. Further investigation is needed to examine the potential of CCR5 antagonists combined with other agents [44]. Using pharmacologic or gene editings to inhibit CCR5 expression protects host cells from viral infection specific for ccr5 [45].

Zinc-finger nucleases were used to modify the gene of $c c r 5$ and $c x c r 4$ in human CD4+ T cells. These modified-ccr5 and cxcr4 cells divided, proliferated, and remained resistant to HIV-1 in vitro. The modified cells were implanted into humanized mice with HIV-1 infection. These ccr5 negative cells were engrafted without major complications and were protected from both CCR5 and CXCR4 specific strains of HIV-1 [45]. Allogeneic forms of CCR5-depleted cells from donors to be transplanted into HIV-positive individuals have immense potential as an alternative therapy. However, there was a limited number of HLA-matched donors. There was only one donor with the CCR5-delta32 homozygous deletion found. Overall, this search failed [46-Hutter, 2011]. Currently, there are CCR5-specific therapies against HIV infection known as maraviroc and vicriviroc being used as cancer therapeutics in clinical trials [31]. Many tumors express CCR5 on the surface, as a result, a combination of therapies are being examined for treating tumors with CCR5 antagonists.

Because the CCR5 coreceptor allows entry of HIV, it has been shown to provide protection against HIV infection with treatment and prevention. Many CCR5 antagonists have been analyzed in clinical trials, but only maraviroc was approved for treating HIV positive patients [44]. The potency and safety of CCR5 antagonists such as maraviroc was evaluated in clinics. Maraviroc and other CCR5 antagonists can prevent HIV transmission, provide an intense HIV treatment, and prevent the rejection of engrafted organ transplants. CCR5 antagonists can increase the potency of rapamycin that downregulates CCR5 co-receptors to reduce the density of CCR5. However, using CCR5 antagonists can cause consequences with West Nile and Tick-borne encephalitis infections [47]. The use of CCR5 antagonists alone or with other agents still requires future and further examination.

Antagonists of CCR5 block HIV entry by binding to CCR5 causing changes in the CCR5 extracellular loops (ECLs). Tilton et al. found that viruses from one patient produced resistance to maraviroc (MVC) during the clinical trial [47]. These MVC resistant viruses were sensitive to other CCR5 antagonists such as vicriviroc and aplaviroc. Resistance to MVC depended on mutations in the V3 loop of the viral envelope (Env) protein and was controlled by more mutations in the V4 loop. After deep sequencing of pretreated plasma with viral RNA, the resistance appeared to have originated from the evolution of a drug bound to CCR5 use regardless of the presence of viral DNA sequences as result of a switch to CXCR4 use [47]. Envs were acquired from this patient before and during MVC administration and treatment that could infect cells with low CCR5 expression levels. There have been previous reports of viral resistance to CCR5 antagonists when drugs modified ECLs of CCR5 for viral entry. CCR5 resistance to many CCR5 antagonists where the viruses specific for the $\mathrm{N}$ terminus with antagonistspecific ECL changes show a small cross-resistance type of characteristic [47].

C-C chemokine receptor types 2 (CCR2) and 5 (CCR5) with their ligands of CCL2 and CCL5, control fibrogenesis through increasing monocyte and macrophage recruitment to tissues and activate hepatic cells. Cenicriviroc (CVC) is an oral drug that is an CCR2 and CCR5 antagonist that provides potent blockades of each two receptors. CVC promotes anti-inflammation and antifibrotic effects. Monocytes and macrophages recruit in vivo in mouse models with thioglycollate-induced peritonitis. CCL2 induces a chemotaxis ex vivo in mouse monocytes. CVCs can cause anti fibrosis that was examined in a thioacetamide-induced mouse model for fibrosis of the liver. CVC lowered monocyte and macrophage 
recruitments in vivo at CVC doses of $\geq 20 \mathrm{mg} / \mathrm{kg} /$ day $(p<0.05)$ [48]. With these doses CVC produced potent antifibrotic effects, reduced collagen deposition $(p<0.05)$, and lowered collagen type 1 proteins with mRNA transcription in three animal models in fibrosis. Using the NASH model, CVC lessened nonalcoholic fatty liver disease score of activity $(p<0.05)$ [48]. CVC showed no effect on body, liver, or kidney weight. CVC increases anti-inflammatory and antifibrotic activity in many animal models for fibrosis that can support testing of human diseases caused by fibrosis.

\section{CONCLUSION}

The aim of this review was to demonstrate the immense impact of CCR5 SNP mutations on viral infection susceptibility, the pathogenesis of chronic conditions, to endorse the increased discovery of more novel CCR5 SNPs, and to show the significant potential of anti-CCR5 therapies that can treat multiple diseased conditions. For example, in the South African black population groups, 39 CCR5 mutations were found. HIV is the most dominant infectious disease in Sub-Saharan Africa where more genetic variability of CCR5 is present. Studying these CCR5 genetic variations can provide and guide the development of antibodies for CCR5 reduced cell surface expression. Genetic variations of CCR5 SNPs such as 2459G>SNP of CCR5, C101X, CCR5 gene position at -2273, and the A to G SNP mutation was found in two South African blacks can be targeted by anti-CCR5 antibodies. However, many CCR5 SNPs have not been reported in studies to demonstrate how these mutations reduce CCR5 expression, lessens disease progression, and controls HIV infection.

Type 2 diabetes (T2D), multiple sclerosis (MS), and asthma is affected by chemokines that initiate and control the movement of immune cells. CCR5, chemokine receptor, binds to RANTES, MIP$1 \alpha$, and MIP-1 $\beta$. NK cells, T lymphocytes, and macrophages used CCR5 chemokine receptors. CCR5 regulates immune cells migration and initiation while immune cells respond to foreighn microbes. The self-antigens of autoimmunity disorders are transported and activated by chemokines binding to CCR5 receptors. Mutations that change the sequence of the CCR5 gene that can cause disorders of the immune system. A 32-base pair deletion $(\triangle 32)$ of CCR5 can change autoimmune disorders and is also related to the pathogenesis of autoimmune disorders [49]. The CCR5- $\Delta 32$ deletion can increase the risk of developing MS, T2D, but not associated with asthma. Possible therapies that can target CCR5 SNPs include: blocking CCR5 to reduce pro-tumor indeed inflammation, control tumorigenic production, gene editing of $c c r 5$ to protect immune cells from HIV-1 infection, allogeneic engraftment of CCR5 depleted immune cells from donors, treatment of tumors with CCR5 antagonists, and an oral drug named cenicriviroc is a CCR2 and CCR5 antagonist that can reduce fibrosis of the liver by lessening proinflammatory responses.

Increased research is required to examine many of the novel mutations and SNPs of CCR5 that affect ligand binding affinity and CCR5 interactions with pathogenic agents. For example, the hCCR5D24 deletion was lacking in many HIV-1 positive mothers and 224 HIV positive infants. A majority of Caucasions do not show this hCCR5D24 deletion. Therefore, the hCCR5D24 deletion is specific to and preserved in the population of East Africa. For individuals homozygous for a wild-type mutation of CCR5 is more prevalent in the Crimean-Congo with patients positive for haemorrhagic fever. Mice that were given a knock-out mutation of CCR5 were protected from Dengue viral infection. The discovery of the hCCR5D24 novel CCR5 SNP was incredible and can accelerate the discovery of more novel CCR5 SNPs. Future research is needed to find additional novel SNPs of CCR5. Flow cytometry is a method that can guide the discovery of novel CCR5 mutations that alter the CCR5 cell surface expression where its expression may not depend on a single mutation. Studying how multiple novel CCR5 SNPS determine the plasticity of the CCR5 structural interaction with pathogenic material may provide additional therapies to treat a diverse array of infectious diseases and chronic health conditions. 


\section{ACKNOWLEDGEMENTS}

Special thanks are given to my mentors. The weekly genetic engineering lab workshops provided and the meetings for the discussion of my future projects helped to increase my understanding of the fundamentals of homologous recombination, microbes, and gene editing.

Author Contributions: T.H., wrote, reviewed, and edited the manuscript.

Funding: The author received no specific funding for this work.

Conflicts of interest: The author declares no conflict of interest.

\section{REFERENCES}

1. Gurdol F, Yurdum LM, Ozturk U, Isbilen E, Cakmakoglu B. Association of the CC chemokine receptor 5 (CCR5) polymorphisms with preeclampsia in Turkish women. Archives of gynecology and obstetrics. 2012 Jul 1;286(1):51-4.

2.Abdolmohammadi R, Azar SS, Khosravi A, Shahbazi M. CCR5 polymorphism as a protective factor for hepatocellular carcinoma in hepatitis B virus-infected Iranian patients. Asian Pacific journal of cancer prevention: APJCP. 2016;17(10):4643.

3.Deval H, Alagarasu K, Mittal M, Srivastava N, Bachal R, Gondhalekar A, Chaudhary U, Chowdhary D, Bondre VP. Association of single nucleotide polymorphisms in TNFA and CCR5 genes with Japanese Encephalitis: A study from an endemic region of North India. Journal of Neuroimmunology. 2019 Nov 15;336:577043.

4.Ferreira-Fernandes H, Santos AC, Motta FJ, Canalle R, Yoshioka FK, Burbano RR, Rey JA, Da Silva $\mathrm{BB}$, Pinto GR. Prevalence of CCR5- $\triangle 32$ and CCR2-V64I polymorphisms in a mixed population from northeastern Brazil. Genetics and Molecular Research. 2015 Jan 1;14(4):11710-8.

5.Zare-Bidaki M, Karimi-Googheri M, Hassanshahi G, Zainodin In, Arababadi MK. The frequency of CCR5 promoter polymorphisms and CCR5 $\Delta 32$ mutation in Iranian populations. Iranian journal of basic medical sciences. 2015 Apr;18(4):312.

6.Nkenfou CN, Mekue LC, Nana CT, Kuiate JR. Distribution of CCR5-Delta32, CCR5 promoter 59029 A/G, CCR2-64I and SDF1-3'A genetic polymorphisms in HIV-1 infected and uninfected patients in the west region of Cameroon. BMC research notes. 2013 Dec 1;6(1):288.

7.Bleul CC, Wu L, Hoxie JA, Springer TA, Mackay CR. The HIV coreceptors CXCR4 and CCR5 are differentially expressed and regulated on human $T$ lymphocytes. Proceedings of the National Academy of Sciences. 1997 Mar 4;94(5):1925-30.

8.Garcia-Perez J, Rueda P, Alcami J, Rognan D, Arenzana-Seisdedos F, Lagane B, Kellenberger E. Allosteric model of maraviroc binding to CC chemokine receptor 5 (CCR5). Journal of Biological Chemistry. 2011 Sep 23;286(38):33409-21. 
9.Joshi A, Punke EB, Sedano M, Beauchamp B, Patel R, Hossenlopp C, Alozie OK, Gupta J, Mukherjee D, Garg H. CCR5 promoter activity correlates with HIV disease progression by regulating CCR5 cell surface expression and CD4 T cell apoptosis. Scientific reports. 2017 Mar 22;7(1):1-1.

10.Mehlotra RK. CCR5 Promoter Polymorphism- 2459G> A: Forgotten or Ignored?. Cells. 2019 Jul;8(7):651.

11.Gornalusse GG, Mummidi S, Gaitan AA, Jimenez F, Ramsuran V, Picton A, Rogers K, Manoharan MS, Avadhanam N, Murthy KK, Martinez H. Epigenetic mechanisms, T-cell activation, and CCR5 genetics interact to regulate T-cell expression of CCR5, the major HIV-1 coreceptor. Proceedings of the National Academy of Sciences. 2015 Aug 25;112(34):E4762-71.

12.Le AQ, Taylor J, Dong W, McCloskey R, Woods C, Danroth R, Hayashi K, Milloy MJ, Poon AF, Brumme ZL. Differential evolution of a CXCR4-using HIV-1 strain in CCR5wt/wt and CCR5 $32 / \Delta 32$ hosts revealed by longitudinal deep sequencing and phylogenetic reconstruction. Scientific reports. 2015 Dec 3;5:17607.

13.Alonzo III F, Kozhaya L, Rawlings SA, Reyes-Robles T, DuMont AL, Myszka DG, Landau NR, Unutmaz D, Torres VJ. CCR5 is a receptor for Staphylococcus aureus leukotoxin ED. Nature. 2013 Jan;493(7430):51-5.

14.Tan Q, Zhu Y, Li J, Chen Z, Han GW, Kufareva I, Li T, Ma L, Fenalti G, Li J, Zhang W. Structure of the CCR5 chemokine receptor-HIV entry inhibitor maraviroc complex. Science. 2013 Sep 20;341(6152):1387-90.

15.Choe H, Farzan M, Sun Y, Sullivan N, Rollins B, Ponath PD, Wu L, Mackay CR, LaRosa G, Newman W, Gerard N. The $\beta$-chemokine receptors CCR3 and CCR5 facilitate infection by primary HIV-1 isolates. Cell. 1996 Jun 28;85(7):1135-48.

16.Barmania F, Potgieter M, Pepper MS. Mutations in CC chemokine receptor type 5 (CCR5) in South African individuals. International Journal of Infectious Diseases. 2013 Dec 1;17(12):e1148-53.

17.Arendt V, Amand M, Iserentant G, Lemaire M, Masquelier C, Ndayisaba GF, Verhofstede C, Karita E, Allen S, Chevigné A, Schmidt JC. Predominance of the heterozygous CCR 5 delta-24 deletion in African individuals resistant to HIV infection might be related to a defect in CCR 5 addressing at the cell surface. Journal of the International AIDS Society. 2019 Sep;22(9):e25384.

18.de Oliveira AP, Bernardo CR, Camargo AV, Ronchi LS, Borim AA, Brandao de Mattos CC, de Campos Junior E, Castiglioni L, Netinho JG, Cavasini CE, Bestetti RB. Genetic susceptibility to cardiac and digestive clinical forms of chronic Chagas disease: involvement of the CCR5 59029 A/G polymorphism. PLoS One. 2015 Nov 23;10(11):e0141847.

19.Sanchooli J, Sanadgol N, Kazemi Arababadi M, Kennedy D. CCR5 plays important roles in hepatitis B infection. Viral immunology. 2014 Feb 1;27(1):2-6.

20.Cao M, Tian Z, Zhang L, Liu R, Guan Q, Jiang J. Effects of CCR5 59029G/A polymorphism on the risk to diabetic nephropathy. Oncotarget. 2017 Dec 5;8(63):106926.

22.Li J, Peng Y, Liu H, Wu Q. The association between CCR5 $\Delta 32$ polymorphism and susceptibility to breast cancer. Oncotarget. 2017 Oct 10;8(47):82796. 
23.Ting KH, Ueng KC, Chiang WL, Chou YE, Yang SF, Wang PH. Relationship of genetic polymorphisms of the chemokine, CCL5, and its receptor, CCR5, with coronary artery disease in Taiwan. EvidenceBased Complementary and Alternative Medicine. 2015 Jan 1;2015.

24.Bagci B, Bagci G, Huzmeli C, Sezgin I, Ozdemir O. Associations of fractalkine receptor (CX3CR1) and CCR5 gene variants with hypertension, diabetes and atherosclerosis in chronic renal failure patients undergoing hemodialysis. International urology and nephrology. 2016 Jul 1;48(7):1163-70.

25.Yahya MJ, Yusoff MJ. Association of CCL2, CCR5, ELMO1, and IL8 polymorphism with diabetic nephropathy in Malaysian type 2 diabetic patients. International journal of chronic diseases. 2019 Jan $1 ; 2019$.

26.Song GG, Kim JH, Lee YH. The chemokine receptor 5 delta32 polymorphism and type 1 diabetes, Behcet's disease, and asthma: a meta-analysis. Immunological investigations. 2014 Feb 1;43(2):123-36.

27.Słomiński B, Ławrynowicz U, Myśliwska J, Ryba-Stanisławowska M, Skrzypkowska M, Brandt A. CCR5- $\triangle 32$ gene polymorphism is associated with retinopathy in patients with type 1 diabetes. Molecular and cellular endocrinology. 2017 Jan 5;439:256-60.

28.Słomiński B, Ławrynowicz U, Ryba-Stanisławowska M, Skrzypkowska M, Myśliwska J, Myśliwiec M. CCR5- $\Delta 32$ polymorphism is a genetic risk factor associated with dyslipidemia in patients with type 1 diabetes. Cytokine. 2019 Feb 1;114:81-5.

29.Didigu CA, Wilen CB, Wang J, Duong J, Secreto AJ, Danet-Desnoyers GA, Riley JL, Gregory PD, June $\mathrm{CH}$, Holmes MC, Doms RW. Simultaneous zinc-finger nuclease editing of the HIV coreceptors ccr5 and cxcr4 protects CD4+ T cells from HIV-1 infection. Blood, The Journal of the American Society of Hematology. 2014 Jan 2;123(1):61-9.

30. Hütter G, Thiel E. Allogeneic transplantation of CCR5-deficient progenitor cells in a patient with HIV infection: an update after 3 years and the search for patient no. 2. Aids. 2011 Jan 14;25(2):273-4.

31.Jiao X, Nawab O, Patel T, Kossenkov AV, Halama N, Jaeger D, Pestell RG. Recent advances targeting CCR5 for cancer and its role in immuno-oncology. Cancer research. 2019 Oct 1;79(19):4801-7.

32.Ishida Y, Kimura A, Kuninaka Y, Inui M, Matsushima K, Mukaida N, Kondo T. Pivotal role of the CCL5/CCR5 interaction for recruitment of endothelial progenitor cells in mouse wound healing. The Journal of clinical investigation. 2012 Feb 1;122(2):711-21.

33. Halama N, Zoernig I, Berthel A, Kahlert C, Klupp F, Suarez-Carmona M, Suetterlin T, Brand K, Krauss J, Lasitschka F, Lerchl T. Tumoral immune cell exploitation in colorectal cancer metastases can be targeted effectively by anti-CCR5 therapy in cancer patients. Cancer cell. 2016 Apr 11;29(4):587-601.

34.Jones KL, Maguire JJ, Davenport AP. Chemokine receptor CCR5: from AIDS to atherosclerosis. British journal of pharmacology. 2011 Apr;162(7):1453-69.

35. Kitade H, Sawamoto K, Nagashimada M, Inoue H, Yamamoto Y, Sai Y, Takamura T, Yamamoto H, Miyamoto KI, Ginsberg HN, Mukaida N. CCR5 plays a critical role in obesity-induced adipose tissue 
inflammation and insulin resistance by regulating both macrophage recruitment and M1/M2 status. Diabetes. 2012 Jul 1;61(7):1680-90.

36.Velasco-Velázquez M, Jiao X, De La Fuente M, Pestell TG, Ertel A, Lisanti MP, Pestell RG. CCR5 antagonists blocks metastasis of basal breast cancer cells. Cancer research. 2012 Aug 1;72(15):3839-50.

37.Schlecker E, Stojanovic A, Eisen C, Quack C, Falk CS, Umansky V, Cerwenka A. Tumor-infiltrating monocytic myeloid-derived suppressor cells mediate CCR5-dependent recruitment of regulatory $T$ cells favoring tumor growth. The Journal of Immunology. 2012 Dec 15;189(12):5602-11.

38.Dobaczewski M, Xia Y, Bujak M, Gonzalez-Quesada C, Frangogiannis NG. CCR5 signaling suppresses inflammation and reduces adverse remodeling of the infarcted heart, mediating recruitment of regulatory T cells. The American journal of pathology. 2010 May 1;176(5):2177-87.

39.Gorgi Y, Sfar I, Jendoubi-Ayed S, Makhlouf M, Romdhane TB, Bardi R, Aouadi H, Abdallah TB, Abderrahim E, Ayed K. Allograft renal rejection and chemokine polymorphism. Saudi Journal of Kidney Diseases and Transplantation. 2011 Jan 1;22(1):18.

40.Sato H, Williams HR, Spagnolo P, Abdallah A, Ahmad T, Orchard TR, Copley SJ, Desai SR, Wells AU, Du Bois RM, Welsh KI. CARD15/NOD2 polymorphisms are associated with severe pulmonary sarcoidosis. European Respiratory Journal. 2010 Feb 1;35(2):324-30.

41.Bedognetti D, Spivey TL, Zhao Y, Uccellini L, Tomei S, Dudley ME, Ascierto ML, De Giorgi V, Liu Q, Delogu LG, Sommariva M. CXCR3/CCR5 pathways in metastatic melanoma patients treated with adoptive therapy and interleukin-2. British journal of cancer. 2013 Oct;109(9):2412-23.

42.Cornu TI, Mussolino C, Bloom K, Cathomen T. Editing CCR5: a novel approach to HIV gene therapy. Gene Therapy for HIV and Chronic Infections. 2015:117-30.

43.Ganczak M, Skonieczna-Żydecka K, Drozd-Dąbrowska M, Adler G. Possible Impact of 190G> a CCR2 and $\triangle 32$ CCR5 mutations on decrease of the HBV vaccine immunogenicity-a preliminary report. International journal of environmental research and public health. 2017 Feb;14(2):166.

44.Gilliam BL, Riedel DJ, Redfield RR. Clinical use of CCR5 inhibitors in HIV and beyond. Journal of translational medicine. $2011 \mathrm{Dec} ; 9(1): 1-4$.

45.Didigu CA, Wilen CB, Wang J, Duong J, Secreto AJ, Danet-Desnoyers GA, Riley JL, Gregory PD, June $\mathrm{CH}$, Holmes MC, Doms RW. Simultaneous zinc-finger nuclease editing of the HIV coreceptors ccr5 and cxcr4 protects CD4+ T cells from HIV-1 infection. Blood, The Journal of the American Society of Hematology. 2014 Jan 2;123(1):61-9.

46. Hütter G, Thiel E. Allogeneic transplantation of CCR5-deficient progenitor cells in a patient with HIV infection: an update after 3 years and the search for patient no. 2. Aids. 2011 Jan 14;25(2):273-4.

47. Tilton JC, Wilen CB, Didigu CA, Sinha R, Harrison JE, Agrawal-Gamse C, Henning EA, Bushman FD, Martin JN, Deeks SG, Doms RW. A maraviroc-resistant HIV-1 with narrow cross-resistance to other CCR5 antagonists depends on both N-terminal and extracellular loop domains of drug-bound CCR5. Journal of virology. 2010 Oct 15;84(20):10863-76. 
48.Lefebvre E, Moyle G, Reshef R, Richman LP, Thompson M, Hong F, Chou HL, Hashiguchi T, Plato C, Poulin D, Richards T. Antifibrotic effects of the dual CCR2/CCR5 antagonist cenicriviroc in animal models of liver and kidney fibrosis. PloS one. 2016 Jun 27;11(6):e0158156.

49.Ghorban K, Dadmanesh M, Hassanshahi G, Momeni M, Zare-Bidaki M, Arababadi MK, Kennedy D. Is the CCR5 $\Delta 32$ mutation associated with immune system-related diseases?. Inflammation. 2013 Jun 1;36(3):633-42.

50.Loetscher P, Uguccioni M, Bordoli L, Baggiolini M, Moser B, Chizzolini C, Dayer JM. CCR5 is characteristic of Th1 lymphocytes. Nature. 1998 Jan;391(6665):344-5. 